\title{
Dual TCR-alpha expression on MAIT cells as a potential confounder of TCR interpretation
}

Sara Suliman ${ }^{1}$, Lars Kjer-Nielsen², Sarah K. Iwany ${ }^{1}$, Kattya Lopez Tamara ${ }^{1,3}$, Liyen Loh ${ }^{2,4}$, Ludivine Grzelak ${ }^{2}$, Katherine Kedzierska², Tonatiuh A. Ocampo ${ }^{1}$, Alexandra J. Corbett ${ }^{2}$, James McCluskey², Jamie Rossjohn,6,7, Segundo R León ${ }^{3}$, Roger Calderon ${ }^{3}$, Leonid Lecca Garcia ${ }^{3}$, Megan B. Murray ${ }^{8}$, D. Branch Moody ${ }^{1}$ and Ildiko Van Rhijn ${ }^{1,9}$.

\#Corresponding Author: Ildiko Van Rhijn, Division of Rheumatology, Inflammation and Immunity, Brigham and Women's Hospital, Boston MA. Email: ivanrhijn@bwh.harvard.edu

Running Title: Dual TCR $\alpha$-expressing MAIT cells

Word Count: 3,498 


\section{Affiliations:}

${ }^{1}$ Division of Rheumatology, Inflammation and Immunity, Brigham and Women's Hospital, Harvard Medical School, Boston, MA, USA

${ }^{2}$ Department of Microbiology and Immunology, University of Melbourne at the Peter Doherty Institute for Infection and Immunity, Melbourne, Victoria, Australia

${ }^{3}$ Socios En Salud Sucursal Peru, Lima, Peru.

${ }^{4}$ Department of Immunology and Microbiology, University of Colorado, Anschutz Medical Campus, Aurora, Aurora, CO, USA

${ }^{5}$ Infection and Immunity Program and The Department of Biochemistry and Molecular Biology, Biomedicine Discovery Institute, Monash University, Clayton, Victoria, Australia

${ }^{6}$ Australian Research Council Centre of Excellence in Advanced Molecular Imaging, Monash University, Clayton, Victoria 3800, Australia.

${ }^{7}$ Institute of Infection and Immunity, Cardiff University School of Medicine, Heath Park, Cardiff CF14 4XN, UK

${ }^{8}$ Department of Global Health and Social Medicine, and Division of Global Health Equity, Brigham and Women's Hospital, Harvard Medical School, Boston, MA, USA

${ }^{9}$ Department of Infectious Diseases and Immunology, Faculty of Veterinary Medicine, Utrecht University, Yalelaan 1, 3584CL Utrecht, The Netherlands 


\section{Abstract (244 words)}

Mucosal-associated invariant T (MAIT) cells are innate-like $\mathrm{T}$ cells that are highly abundant in human blood and tissues. Most MAIT cells have an invariant T cell receptor (TCR) $\alpha$ chain that uses TRAV1-2 joined to TRAJ33/20/12 and recognize metabolites from bacterial riboflavin synthesis bound to the antigen-presenting molecule, MR1. Recently, our attempts to identify alternative MR1-presented antigens led to the discovery of rare MR1-restricted T cells with non-TRAV1-2 TCRs. Because altered antigen specificity is likely to lead to altered affinity for the most potent known antigen, 5-(2-oxopropylideneamino)-6-D-ribitylaminouracil (5-OP-RU), we performed bulk TCR $\alpha$ and $\beta$ chain sequencing, and single cell-based paired TCR sequencing, on T cells that bound the MR1-5-OP-RU tetramer, but with differing intensities. Bulk sequencing showed that use of $V$ genes other than TRAV1-2 was enriched among MR1-5-OP-RU tetramer ${ }^{\text {low }}$ cells. Whereas we initially interpreted these as diverse MR1-restricted TCRs, single cell TCR sequencing revealed that cells expressing atypical TCR $\alpha$ chains also co-expressed an invariant MAIT TCR $\alpha$ chain. Transfection of each non-TRAV1-2 TCR $\alpha$ chain with the TCR $\beta$ chain from the same cell demonstrated that the non-TRAV1-2 TCR did not bind the MR1-5-OP-RU tetramer. Thus, dual TCR $\alpha$ chain expression in human T cells and competition for the endogenous $\beta$ chain explains the existence of some MR1-5-OP-RU tetramer ${ }^{\text {low }}$ T cells. The discovery of simultaneous expression of canonical and non-canonical TCRs on the same T cell means that claims of roles for non-TRAV1-2 TCR in MR1 response must be validated by TCR transfer-based confirmation of antigen specificity. 


\section{Introduction}

Adaptive cellular immunity relies on recombination of the T cell receptor (TCR)- $\beta$ (TRB), TCR- $\gamma(T R G)$, TCR- $\alpha$ and TCR- $\delta$ (TRA/TRD) genomic loci during T cell development in the thymus ${ }^{1}$. Remarkable TCR diversity is achieved by combinatorial usage of genome-encoded variable $(\mathrm{V})$, diversity $(\mathrm{D})$, and joining $(\mathrm{J})$ genes, and addition of intervening non-templated $(\mathrm{N})$ nucleotides $^{2}$. Many T cells recognize peptide antigens in the context of highly polymorphic human leukocyte antigen (HLA) molecules ${ }^{3}$. In parallel, some T cells bind non-peptide antigens presented by non-MHC-encoded antigen-presenting molecules, including the MHC-related protein 1 (MR1) and cluster of differentiation (CD)1 proteins (reviewed in ${ }^{4,5}$ ). Unlike MHC, CD1 and MR1 proteins are almost monomorphic ${ }^{6}$, and consequently CD1- and MR1-reactive T cells tend to express characteristic TCR motifs, shared by many individuals irrespective of their HLA haplotypes ${ }^{7}$. These invariant TCR motifs $^{7}$ recognize unique antigen classes, including pathogen-derived mycobacterial lipids for $C D 1 b^{8}, \alpha$-galactosyl ceramides for $C D 1 d^{9}$ and metabolites from active bacterial biosynthetic enzymes for MR ${ }^{10}$. These invariant TCRs are thought to have co-evolved with cognate nonclassical antigen-presenting molecules in different species ${ }^{11}$.

Due to their potential to elicit generalizable population-level immune responses, donorunrestricted T cells (DURTs), and the antigens they recognize, are attractive targets of vaccination against microbes like Mycobacterium tuberculosis $(M t b)^{12}$. In particular, mucosal-associated invariant T (MAIT) cells, which recognize antigens presented by MR1, are attractive candidates due to their abundance in the blood ${ }^{13}$, their high reactivity against several bacterial infections ${ }^{14,15}$ 16, 17 , and their documented roles in vaccination ${ }^{18,19}$. MR1 tetramers bind directly to TCRs and allow for unequivocal identification of MAIT cells and more diverse MR1-restricted $\alpha \beta^{20}$ and $\gamma \delta^{21} \mathrm{~T}$ cells, and provide a unique opportunity to identify novel TCR rearrangements and antigen specificities $^{22}$. Human MAIT TCR $\alpha$ chains display a characteristic complementarity-determining region $(\mathrm{CDR} 3 \alpha)$ formed by a rearrangement between TRAV1-2 and TRAJ33, or sometimes 
TRAJ12 or TRAJ20, with few non-template encoded (N)-nucleotides ${ }^{22,23,24}$, and a biased preference for some TRB genes ${ }^{23,25,26}$. Diversity in TRB gene usage in MAIT cells is potentially associated with recognition of different microbes ${ }^{25,27,28,29}$ or different ligands ${ }^{30}$. These canonical MAIT cells have a preferred specificity for 5-(2-oxopropylideneamino)-6-D-ribitylaminouracil (5OP-RU) over 6-formylpterin (6-FP) $)^{10,31,32}$. Whereas TCR conservation, especially 'canonical' TRAV1-2 usage has been considered a key defining feature of human MAIT cells for decades, a new direction in the field has resulted from identification of 'non-canonical' TRAV1-2-negative $\left(\mathrm{TRAV} 1-2^{-}\right)$and $\gamma \delta \mathrm{T}$ cells $^{21}$ that recognize MR1 and suggested to have unique antigen specificities $^{20,33,34,35,36,37}$. MAIT cells have broadly reported roles in infection ${ }^{17}$, cancer $^{38}$, and autoimmunity ${ }^{39}$. Hence, defining MAIT TCR motifs can be used to infer pathogenic and protective TCR clonotypes relevant to immunodiagnosis or vaccination.

Several new technologies and algorithms for high-dimensional TCR sequencing analysis have successfully identified clonally expanded populations of antigen-specific T cells, and their TCR motifs among large numbers of blood- and tissue-derived T-cells ${ }^{40,41,42,43}$. These sequencing technologies derive TCR sequences either from single cells, which identify paired TCR $\alpha$ and TCR $\beta^{44,45}$, or bulk genomic ${ }^{46}$ or transcriptomic sequencing data ${ }^{41,47}$. In this study, we sought to use MR1 tetramers and high throughput TCR sequencing to identify non-canonical TCR patterns. We observed MAIT cell populations with differing binding intensities to the 5-OP-RUloaded MR1 tetramers. We hypothesized that MAIT cells with lower MR1-tetramer binding intensities would reveal unique TCR motifs consistent with lower preferential binding to the 5-OPRU/MR1 antigen complex. Consistently, we detected an enrichment of TRAV1-2- TCRs in MR1 tetramer ${ }^{+}$MAIT cells, especially those with lower MR1-tetramer intensity. However, detailed TCR gene transfer studies revealed that the lower tetramer binding was explained by dual expression of canonical and non-canonical TCR $\alpha$ chains in the same TRAV1-2+ clonally expanded MAIT cells, as opposed to a single non-canonical TCR with lower affinity for MR1-5-OP-RU. Dual TCR 
expression previously observed in HLA-restricted ${ }^{48}$ and CD1d-reactive $\mathrm{T}_{\text {cells }}{ }^{49}$, but takes on special importance in the MAIT cell system because it can confound the assignment of noncanonical TCRs for MR1 specificity. These data establish the need to validate the antigen specificity of newly-described TCR motifs from large-dimensional sequencing platforms by TCR gene transfer and other alternative techniques ${ }^{50}$. 


\section{Materials and Methods}

\section{Human participants}

Lima, Peru: We recruited Peruvian participants with active TB disease, or asymptomatic household contacts of TB cases with positive or negative QuantiFERON TB Gold-In-tube test results from Lima, Peru, as described previously ${ }^{51,52}$. The Institutional Review Board of the Harvard Faculty of Medicine and Partners HealthCare (protocol number IRB16-1173), and the Institutional Committee of Ethics in Research of the Peruvian Institutes of Health approved this study protocol. All adult study participants and parents and/or legal guardians of minors provided informed consent, while minors provided assent. The protocol is approved by the Institutional Review Board of Harvard Faculty of Medicine and Partners HealthCare, and Institutional Committee of Ethics in Research of the Peruvian Institutes of Health.

Boston, USA: We obtained de-identified leukoreduction filters (leukopak) samples from healthy blood bank donors through the Brigham and Women's Hospital Specimen Bank, as approved by the Institutional Review Board of Partners HealthCare.

Tennessee, Memphis: Peripheral blood mononuclear cell (PBMC) samples were obtained from healthy children, adult, and elderly donors from St. Jude Children's Research Hospital (XPD12089 IIBANK and 1545216.1).

Melbourne, Australia: Spleen (SP) lymphoid tissues were collected from deceased donors, whose mortality was caused by conditions other than influenza (DonateLife, Australia), after written informed consent was given by next of $\mathrm{kin}^{53}$. The University of Melbourne Human Ethics Committee approved experiments (identification numbers 1443389.4, 1955465 and 1545216.1). 


\section{Flow cytometry analysis}

The protocol and primary analysis of Peruvian samples by flow cytometry was reported previously ${ }^{51}$. MR1 monomers were obtained from The University of Melbourne, Australia ${ }^{10,22}$, and used to generate tetramers in Boston as previously described ${ }^{51}$. For HEK293T cell validation experiments, we used MR1 tetramers obtained from the National Institutes of Health $(\mathrm{NIH})$ Tetramer Core facility.

\section{Genomic bulk TCR sequencing (Adaptive Biotechnologies, Seattle)}

For TCR sequencing from genomic templates, 3900 MR1 tetramer ${ }^{\text {hi }}$ and 4500 MR1 tetramer $^{\text {int }}$ cells were doubly sorted from PBMC samples from Peruvian donor 58-1 after 14 days of polyclonal T cell expansion. For expansion, $10^{6}$ cells were cultured with $25 \times 10^{6}$ irradiated allogeneic PBMC, $5 \times 10^{6}$ irradiated allogeneic Epstein Barr Virus transformed B cells, $30 \mathrm{ng} / \mathrm{ml}$ anti-CD3 monoclonal antibody (clone OKT3) for 14-16 days, in the presence of $1 \mathrm{ng} / \mathrm{ml}$ interleukin-2 (IL-2) $)^{52}$. PBMC samples from healthy Boston blood bank donors LP1 and CO2 were not expanded before double cell sorting. Cell numbers obtained from the sorted tetramer ${ }^{\text {hi }}$, tetramer $^{\text {int }}$, and tetramer ${ }^{\text {low }}$ populations were $2000,5800,3100$, respectively, for LP1 and 1100 , 4000, and 2300, respectively, for CO2. High-throughput TCR sequencing and assignment of V and $\mathrm{J}$ genes was performed for the TCR $\beta$ locus and the TCR $\alpha \delta$ locus (Adaptive Biotechnologies, Seattle, WA) using a multiplex PCR approach on genomic DNA isolated from sorted T cells using the Qiagen QIAamp DNA Mini Kit, followed by Illumina high-throughput sequencing ${ }^{46}$.

\section{Sorted single cell paired TCR sequencing}

Single-cell TCR sequencing was adapted from a previously published protocol ${ }^{41}$. Briefly, single MR1-tetramer-binding cells from Peruvian participant 7-3 and blood bank donors 702A and 703A were sorted into 96-well plate coated with Vapor-Lock (Qiagen) containing Iscript cDNA synthesis 
mixture (Bio-Rad) and $0.1 \%$ triton X-100 for direct cell lysis. Reverse transcription was performed in a thermocycler $\left(25^{\circ} \mathrm{C}\right.$ for $5^{\prime}, 42^{\circ} \mathrm{C}$ for $30^{\prime}, 80^{\circ} \mathrm{C}$ for $\left.5^{\prime}\right)$. Subsequently, cDNA samples were amplified in a nested PCR reaction using Denville Choice Taq Polymerase (Thomas Scientific), using previously described primers ${ }^{41}$. Briefly, the first external reaction contained a mixture of all TCR $\alpha$ and TCR $\beta$ forward primers, combined at $1 \mu \mathrm{M}$ each, and reverse TRAC and TRBC primers at $10 \mu \mathrm{M}$ each: $95^{\circ} \mathrm{C}$ for $2^{\prime}, 35$ cycles of $\left(95^{\circ} \mathrm{C}\right.$ for $20^{\prime \prime}, 50^{\circ} \mathrm{C}$ for $20^{\prime \prime}, 72^{\circ} \mathrm{C}$ for $\left.45^{\prime \prime}\right)$, and $72^{\circ} \mathrm{C}$ for 7'. A second internal PCR reaction used a mix of TCR $\alpha$ forward primers at $1 \mu \mathrm{M}$ each with a reverse internal TRAC primer at $10 \mu \mathrm{M}$, or a mix of TCR $\beta$ forward primers and reverse TRBC primer, separately at cycling conditions: $95^{\circ} \mathrm{C}$ for $2^{\prime}, 35$ cycles of $\left(95^{\circ} \mathrm{C}\right.$ for $20^{\prime \prime}, 56^{\circ} \mathrm{C}$ for $20^{\prime \prime}, 72^{\circ} \mathrm{C}$ for $45^{\prime \prime}$ ), and $72^{\circ} \mathrm{C}$ for $7^{\prime}$ using previously described primers ${ }^{41}$. Amplicons were analyzed on an agarose gel, and bands were excised using a UV lamp and purified using the QIAquick Gel Extraction Kit (Qiagen) then sent for Sanger sequencing (Genewiz). Sequences were reversecomplemented and analyzed using 4Peaks software and mapped to the reference sequences for the genome-encoded $V$ and $\mathrm{J}$ segments for both the TCR $\alpha$ and TCR $\beta$ genes on the ImMunoGeneTics (IMGT) information system database. The unmapped sequences were considered $\mathrm{N}$-nucleotides, and/or $\mathrm{D} \beta$ segments for TCR $\beta$ to determine the complementaritydetermining region (CDR)-3. CDR3 $\alpha$ and CDR3 $\beta$ amino acid sequences were predicted by in silico translation, showing productive in-frame rearrangements, using the online ExPASy translate tool (https://web.expasy.org/translate/).

For Australian samples, single MR1-5-OP-RU-tetramer ${ }^{+}$TRAV1-2+ PBMCs from healthy donors and spleen tissues were sorted using a FACSAria (BD Biosciences) into 96-well plates. Paired CDR $3 \alpha \beta$ regions were determined using multiplex-nested reverse transcriptase PCR before sequencing of TCR $\alpha$ and TCR $\beta$ products, as previously described ${ }^{41,54}$, and reported ${ }^{55}$. For paired 
TCR $\alpha \beta$ analyses, sequences were parsed into the IMGT/HighV-QUEST web-based tool using TCRBlast1 (kindly provided by Paul Thomas and Matthew Caverley), to determine V(D)J regions.

\section{TCR transfection assay}

Synthetic TCR $\alpha$ and TCR $\beta$ sequences (Genewiz) from MR1 tetramer-binding sorted single T cells, separated by self-cleaving Picornavirus 2A (P2A)-linker sequence (GGATCCGGCGCCACCAATTTCTCGCTGCTTAAGCAGGCCGGCGACGTCGAAGAGAACCC CGGGCCCATG), were cloned into a GFP-containing pMIG vector using standard restriction digestion and cloning procedures. Human embryonic kidney (HEK293T) cells were cultured overnight on a 6-well plate containing $4 \mathrm{~mL}$ of Dulbecco's Modified Eagle Medium-10 media supplemented with $10 \%$ fetal bovine serum and penicillin-streptomycin at $37^{\circ} \mathrm{C}$, and were subsequently co-transfected with the pMIG-TCR and pMIG-CD3 plasmid ${ }^{56}$ using FuGENE HD transfection reagent (Promega). Transfected HEK293-T cells were analyzed for tetramer binding by flow cytometry $48-72$ hours following transfection. Antibodies used to stain transfected 293T cells were Brilliant Violet 421-conjugated anti-human CD3 antibody (Biolegend) and phycoerythrin (PE)-conjugated anti-human TCR $\alpha \beta$ antibody (BD BioSciences). 


\section{Results}

During a quantitative study of MAIT cells in a Peruvian TB cohort ${ }^{51}$, we observed MAIT cell populations with variable staining intensities for the 5-OP-RU-loaded MR1 tetramer (Figure 1A). This phenomenon was observed in participants with and without evidence for Mtb infection and did not seem to be correlated with TB disease. Whereas canonical MAIT TCRs typically show high affinity for MR1-5-OP-RU, we hypothesized that MAIT cells with lower tetramer staining intensity may reflect different and variable TCR motifs, consistent with their lower affinities to the MR1-5-OP-RU complex. To define TCR gene usage in high, intermediate and low staining populations, we sorted MAIT cell populations with different MR1-tetramer staining intensities and performed bulk TCR $\alpha$ and TCR $\beta$ sequencing from genomic DNA and subsequent $\mathrm{V}$ - and J-gene assignment of rearranged genes. Subsequently, we sorted MAIT cell populations from one Peruvian sample (58-1) after polyclonal T cell expansion, and from two random Boston blood bank donors (LP1 and CO2) without expansion (Figure 1B). The populations were sorted based on MR1-tetramer fluorescence intensities and re-sorted prior to sequencing to ensure purity and preservation of MR1-tetramer binding levels (Supplementary Figure 1). Regardless of the source of PBMCs, we saw similar patterns with TRAV1-2 TCRs in brightly staining cells, and TCR $\alpha$ V-genes other than V $\alpha 7.2$ (TRAV1-2) were enriched in sorted MAIT populations with low and intermediate MR1-tetramer staining (Figure 1B-C). This pattern of atypical TRAV gene usage in MAIT cells with lower MR1-tetramer binding relative to MAIT cells with high MR1-tetramer staining was observed even after discarding unproductive TCR $\alpha$ chains (Supplementary Table 1). Frequencies of TRAV1-2- MAIT cells in blood did not differ by TB status in Peruvian samples (Kruskal-Wallis: $p=0.75$; Figure 1D). TRAV1-2- MAIT in these samples (Figure 1B-C) were similar to frequencies previously reported in other populations ${ }^{20}$ representing a minority of $\mathrm{T}$ cells (0.6-40\%) but they were potentially biologically significant because TCR $\alpha$ diversity diverges from the conventional understanding of MAIT cell function. 
We sought to explain the discrepancy between the low frequencies of TRAV1-2- MAIT cells as determined by flow cytometry (Figure 1D), and the higher frequencies of TRAV1-2- TCR $\alpha$ chain sequences identified in sorted MAIT cells, as determined by bulk TCR sequencing (Figure 1C). Hence, we sorted single cells from populations with different MR1-tetramer binding levels from one Peruvian participant, where we detected three clear MR1-tetramer binding levels (MR1tetramer $^{\text {high }}$, MR1-tetramer ${ }^{\text {int }}$, and MR1-tetramer ${ }^{\text {low }}$ ), and applied a previously described nested PCR protocol to cDNA amplified from each single cell ${ }^{41}$ to determine the sequences of paired TCR $\alpha$ and TCR $\beta$ chains (Figure 2A). Non-TRAV1-2 TCR $\alpha$ gene usage was enriched in populations with lower MR1-tetramer binding, with 15/40 (37.5\%) of the MR1-tetramer ${ }^{\text {int }}$ cells using TRAV16 and identical CDR3 $\alpha$ nucleotide sequences, and 14/34 (41.2\%) of the MR1tetramer $^{\text {low }}$ cells using TRAV5, of which 13 had identical CDR3 $\alpha$ nucleotide sequences, suggesting clonal expansion in vivo (Figure 2A and Supplementary Tables 2 and 3). Similarly, we detected TRAV1-2- TCRs from single cell-sorted MR1-tetramer ${ }^{\text {low }}$ populations from two healthy blood bank donors: $1 / 33(3 \%)$ and $8 / 48(16.7 \%)$, but none in MR1-tetramer ${ }^{\text {high }}$ counterparts (Figure 2B). Furthermore, the atypical TRAJ33- joining regions were seen more frequently in low MR1 tetramer staining cells. Overall, these patterns from oligoclonal T cells (Figure 2) matched those of polyclonal T cells (Figure 1) and demonstrated more non-canonical gene usage in TCRs among low MR1 tetramer staining T cells.

To validate the MR1-reactivity of these putative MAIT TCRs, we co-transfected human embryonic kidney (HEK293T) cells with pMIG vectors expressing CD3 and the paired TCR $\alpha$ and TCR $\beta$ sequences derived from three clones with non-TRAV1-2 TCR sequences (Figure 3), which showed clear clonal expansion in samples analyzed with bulk (Figure 1B) or single cell (Figure 2A) TCR sequencing methods. Next, we measured TCR binding to the 5-OP-RU-loaded MR1 tetramer (Figure 3). We also transfected TCR $\alpha$ and TCR $\beta$ from a canonical MAIT TCR (TRAV12-TRAJ33) identified in the bulk-sorted MR1-tetramer ${ }^{\text {high }}$ cells as a positive control (Figure 3). Co- 
transfected HEK293T cells co-expressed CD3 and TCR $\alpha \beta$ on the cell surface (Figure 4, left). The 5-OP-RU-loaded MR1-tetramer, but not the MR1 tetramer loaded with the non-agonist 6-FPloaded MR1 tetramer, stained $\mathrm{CD}^{+}$cells from HEK293T cells transfected with the TRAV1-2 ${ }^{+}$ TCR, as expected. However, the MR1 tetramers, loaded with either 6-FP or 5-OP-RU, did not bind cells expressing the TRAV1-2- TCRs identified in MR1-tetramer ${ }^{\text {low }}$ and MR1-tetramer ${ }^{\text {int }}$ populations (Figure 4), despite the original detection of these TCR sequences in MR1-tetramerbinding cells (Figures 1 and 2).

To explain the lack of binding between these TRAV1-2- TCRs and5-OP-RU-loaded MR1, we took a closer look at the TCR $\beta$ sequences. Unexpectedly, a single TCR $\beta$ sequence consisting of TRBV24-1-TRBJ2-5 with a unique CDR3 nucleotide sequence was detected in 10 out of the 15 TRAV $16^{+}$single cells (Supplementary Table 3). Interestingly, the same TCR $\beta$ nucleotide sequence (TRBV24-1-TRBJ2-5) was paired with the canonical MAIT TCR $\alpha$ TRAV1-2-TRAJ33 in 3 wells (Supplementary Table 3). Because the PCR reactions were performed in multiplex format, we hypothesized that this particular T cell clone expressed two different, functional TCR $\alpha$ chains, but that only one of the PCR products dominated the PCR reaction. Hence, to resolve the discrepancy, we re-amplified the templates that initially gave rise to a TRAV16-TRAJ11 PCR products, using only the TRAV1-specific forward primer, which captures the TCR $\alpha$ variable genes TRAV1-1 and TRAV1-2 only, as previously described ${ }^{41}$. Using this approach, 10 out of the 15 templates initially giving rise to TRAV16-TRAJ11 sequences now gave rise to a PCR product that resulted in identical TRAV1-2-TRAJ33 sequences and paired with the same TRBV24-1-TRBJ2-5 TCR $\beta$ (Supplementary Table 3). Whereas we initially interpreted these results as non-canonical TCRs binding to MR1, the data were more consistent with clonal expansion of a T cell coexpressing one TCR $\beta$ chain, a TRAV1-2 ${ }^{+}$invariant MAIT TCR $\alpha$ chain, and an additional, noncanonical TCR $\alpha$ chain. If only the canonical TCR $\alpha$ chain binds MR1, the lower tetramer binding 
of these TCRs could be caused by competition of two different TCR $\alpha$ chains with the same TCR $\beta$ chain (TRBV24-1-TRBJ2-5), analogous to what has been described for NKT cells ${ }^{49}$.

Finally, to reproduce our finding of dual TCR $\alpha$ expression on MAIT cells in an independent experiment, we analyzed paired TCR sequences in MR1-tetramer-binding cells from different blood donors ${ }^{55}$. Although in this experiment we sorted all MR1 tetramer-binding T cells, including the MR1-tetramer ${ }^{\text {high }}$ ones, we identified cells that co-expressed the canonical invariant TRAV1$2^{+}$TCR $\alpha$ chain with a TRAV1-2 ${ }^{-} \alpha$ chain in PBMC samples from donors of different ages, as well as healthy spleen tissues of deceased donors (Figure 5). Collectively, our study suggests that dual-TCR $\alpha$ expression is common among MR1-tetramer-binding MAIT cells in different human populations, tissue types and disease states. 


\section{Discussion:}

In this study, we hypothesized that TCRs with decreased affinity for MR1-5-OP-RU would reveal new TCR motifs that may prefer MR1 ligands other than 5-OP-RU or correlate with TB disease. Our hypothesis was motivated by the reported expansion of diverse MAIT cell clonotypes following Salmonella challenge of humans in individuals who progress to disease ${ }^{57}$, and the discovery of new antigen classes derived from the related mycobacterium M. smegmatis ${ }^{28}$. However, our search for new TCR motifs based on differential binding to the 5-OP-RU-loaded MR1 tetramer was confounded by the co-expression of two TCR $\alpha$ chains in the same T cell. The phenomenon of dual TCR $\alpha$ co-expression has been previously described for MHC-restricted ${ }^{58,}$ 59 and CD1d-restricted ${ }^{49} \mathrm{~T}$ cell subsets. Unlike the TCR $\beta$ locus, the TCR $\alpha$ counterpart is not subject to strict allelic exclusion, so dual TCR $\alpha$ expression is more common ${ }^{60,61}$. TCR $\alpha$ recombination is also known to occur simultaneously on both alleles to maximize productive $\mathrm{TCR} \alpha \beta$ recombination and diversity in the TCR repertoire ${ }^{62}$.

The simplest explanation for the lower MR1-tetramer staining, which is also supported by these reports of dual TCR $\alpha$ chains in other systems, is that the canonical MAIT TCR binds to MR1, but the competition of the two TCR $\alpha$ chains to pair with the same pool of available TCR $\beta$ chains reduces the MR1-tetramer-binding intensity by reducing functional TCR expression on the cell surface. Hence, the hypothesis that these TCRs displayed preferential affinity to different MR1 antigens was not supported by the data. Importantly, our data point to a potentially common artifact in interpreting TCR $\alpha$ sequences, particularly from high-dimensional sequencing data ${ }^{63}$. Since research focuses on identifying TCR motifs and antigen specificities of non-MHC-restricted DURT cells, including MAIT cells, new TCR motifs require systematic validation for MR1specificity through TCR transfer, especially in light of the reported low frequency of TRAV1-2MAIT cells ${ }^{13,20,33,34}$. 
We detected dual TCRs or lower tetramer staining in multiple donors studied with different methods in two laboratories. These unexpectedly common observations suggest that $\mathrm{T}$ cells with invariant TCR $\alpha$ chains may even have a higher propensity for expression of two TCR $\alpha$ chains compared to conventional MHC-restricted T cells. Several known aspects of conserved TCR gene usage on MAIT cells are consistent with this hypothesis. Firstly, innate T cells, including MAIT ${ }^{24}$, ${ }^{64}$, type I NKT cells ${ }^{65}$, and germline-encoded mycolyl lipid-reactive (GEM) T cells ${ }^{66}$, express TCRs that mostly consist of genome-encoded segments, and few $\mathrm{N}$ nucleotides ${ }^{7}, 67$. TCR $\alpha$ recombination starts from the proximal $\mathrm{V} \alpha$ and $\mathrm{J} \alpha$ genes, and proceeds outwardly towards distal $\mathrm{V} \alpha$ and $\mathrm{J} \alpha$ segments until a productive rearrangement occurs or the cell undergoes apoptosis ${ }^{2}$. TRAV1-2 is the second most distal TCR V $\alpha$ gene, located near the 5' end of the TRA/D locus. The reliance of many invariant T cells on distal TCR $\alpha$ rearrangements involving TRAV1-2 raises the possibility that their thymic progenitors had extended survival windows during the $C D 4^{+} \mathrm{CD} 8^{+}$ double positive (DP) thymocyte stage ${ }^{68}$, when $\mathrm{TCR} \alpha$ recombination took place. However, this hypothesis warrants additional studies.

Prior reports suggest that dual TCR co-expression, i.e., two TCR $\alpha$ chains sharing a single TCR $\beta$ chain, could shelter one TCR $\alpha \beta$ pair from thymic negative selection and consequently increase the propensity for autoimmunity, as reported in mice ${ }^{48,69,70}$ and humans ${ }^{71,72}$. The second reason for a higher likelihood of MAIT and NKT cells to express two TCR $\alpha$ chains is that both subsets have been reported to undergo selection on hematopoietic cells, namely other DP thymocytes, expressing MR1 $1^{73,74,75}$ or $C D 1 d^{76}$, respectively, as opposed to thymic epithelial cells on which MHC-restricted cells are selected ${ }^{77}$. Therefore, selection of MAIT and NKT cell progenitors in separate niches from where strongly autoreactive MHC-restricted cells are often eliminated may render them more likely to escape negative selection despite the potential autoreactivity of the second TCR $\alpha \beta$ receptor. 
We restricted the analysis in this study to MR1-tetramer-binding MAIT cells, with the aim of identifying unique MAIT TCR motifs, and potentially novel antigenic specificities, as recently described $^{20,28,33,34,35}$. To our knowledge, a systematic analysis of the propensities of MHCrestricted T cells and DURTs for expression of dual TCR $\alpha$ chains has not been formally conducted. While our analyses were not intended to directly compare the frequency of dual TCR $\alpha$ expression in donor-unrestricted (innate-like) and MHC-restricted T cells, our study calls for caution when identifying new TCR motifs, particularly in DURTs. These DURTs have unique rules for recognition of non-peptide antigens and antigen-presenting molecules ${ }^{78}$, and hence, functional validation of new TCR motifs is fundamental to this growing field. Collectively, our findings support that TRAV1-2 is the dominant TCR $\alpha$ gene used for recognition of MR1-5-OP-RU, consistent with the reported low frequency of alternative MAIT TCR $\alpha$ V-genes ${ }^{13,20,33}$. 
Figure legends:

Figure 1: TRAV1-2 ${ }^{-}$TCR sequences are enriched in MAIT cells with lower MR1 tetramer staining intensities

(A) Three examples of variable MR1 tetramer staining intensities by flow cytometry in pre-gated T lymphocytes in samples from uninfected, latent, and active TB participants.

(B) Gating strategy for bulk-sorted MAIT cells with different 5-OP-RU-loaded MR1 tetramer staining intensities is shown. The pie charts depict distribution of TCR $\alpha$ gene usage from the different populations.

(C) Gating strategy to identify TRAV1-2- MAIT cells among all MR1-tetramer-binding cells is shown.

(D) Proportions of TRAV1-2- MAIT cells among all MR1-tetramer-binding cells in the Peruvian samples from healthy participants who are either uninfected or infected with Mycobacterium tuberculosis, and active TB patients are shown. Error bars denote medians and interquartile ranges.

Figure 2: Single-cell sorted MAIT cells also show enrichment of TRAV1-2-negative TCR sequences

(A) Gating strategy shows single cell-sorted MAIT cells with different 5-OP-RU-loaded MR1 tetramer staining intensities in Peruvian latent sample no. 7-3. The pie charts depict distribution of TCR $\alpha$ gene usage from the different sorted populations.

(B) Pie charts showing distribution of TCR $\alpha$ V-gene usage in single-cell-sorted MR1-tetramer ${ }^{\text {high }}$ and MR1-tetramer ${ }^{\text {low }} \mathrm{T}$ cells from two additional healthy blood bank donors. 
Figure 3: T-cell receptor sequences for additional validation by HEK293T cell transfection experiments. * Templates from this reaction were re-amplified using TRAV1 forward primer only with TRAC reverse primer (Figure 4).

Figure 4: HEK293T cells transfected with non-TRAV1-2 TCRs from MR1-tetramer-sorted cells do not bind MR1

The plots show flow cytometry of human embryonic kidney (HEK) 293-T cells co-transfected with pMIG vectors expressing CD3 and paired TCR $\alpha$ and TCR $\beta$ sequences from TCR sequences identified in sorted MR1-tetramer-binding populations with different MR1-binding intensities. The left panel shows gating of CD3- and CD3+ populations used to derive the overlayed histograms are gated on CD3- (grey) and CD3+ (black).

Figure 5: Examples of dual TCR $\alpha$-expressing MAIT cell clones detected in different sample types. Codes: $\mathrm{CH}$ (Child), AD (Adult), ED (Elderly), SP(Spleen). 


\section{Acknowledgements:}

This study was supported by the National Institutes of Health (NIH) TB Research Unit Network, Grant U19 Al111224-01. SS received the Justice, Equity, Diversity and Inclusion (JEDI) award, which provided free language editorial service on the manuscript. AJC is supported by a Future Fellowship (FT160100083) from the Australian Research Council, an Investigator Grant from the National Health and Medical Research Council of Australia (1193745) and a Dame Kate Campbell Fellowship from the University of Melbourne. The content is solely the responsibility of the authors and does not necessarily represent the official views of the National Institutes of Health. KK was supported by the NHMRC Leadership Investigator Grant (\#1173871).

\section{Declaration:}

LKN, AJC, JMcC, and JR are named co-inventors on patents describing MR1 tetramers. The MR1 tetramer technology was developed jointly by Prof. James McCluskey, Prof. Jamie Rossjohn, and Prof. David Fairlie, and the material was produced by the NIH Tetramer Core Facility as permitted to be distributed by the University of Melbourne. 


\section{References:}

1. Krangel, M.S. Mechanics of T cell receptor gene rearrangement. Curr Opin Immunol 21, 133-139 (2009).

2. Haynes, M.R. \& Wu, G.E. Evolution of the variable gene segments and recombination signal sequences of the human T-cell receptor alpha/delta locus. Immunogenetics 56, 470-479 (2004).

3. Zinkernagel, R.M. \& Doherty, P.C. Restriction of in vitro T cell-mediated cytotoxicity in lymphocytic choriomeningitis within a syngeneic or semiallogeneic system. Nature 248, 701-702 (1974).

4. Godfrey, D.I., Uldrich, A.P., McCluskey, J., Rossjohn, J. \& Moody, D.B. The burgeoning family of unconventional T cells. Nat Immunol 16, 1114-1123 (2015).

5. Huang, S. \& Moody, D.B. Donor-unrestricted T cells in the human CD1 system. Immunogenetics 68, 577-596 (2016).

6. Reinink, P. \& Van Rhijn, I. Mammalian CD1 and MR1 genes. Immunogenetics 68, 515523 (2016).

7. Van Rhijn, I. \& Moody, D.B. Donor Unrestricted T Cells: A Shared Human T Cell Response. J Immunol 195, 1927-1932 (2015).

8. Van Rhijn, I. \& Moody, D.B. CD1 and mycobacterial lipids activate human T cells. Immunol Rev 264, 138-153 (2015).

9. Spada, F.M., Koezuka, Y. \& Porcelli, S.A. CD1d-restricted recognition of synthetic glycolipid antigens by human natural killer T cells. J Exp Med 188, 1529-1534 (1998).

10. Corbett, A.J. et al. T-cell activation by transitory neo-antigens derived from distinct microbial pathways. Nature 509, 361-365 (2014).

11. Boudinot, P. et al. Restricting nonclassical MHC genes coevolve with TRAV genes used by innate-like T cells in mammals. Proc Natl Acad Sci U S A 113, E2983-2992 (2016).

12. Joosten, S.A. et al. Harnessing donor unrestricted T-cells for new vaccines against tuberculosis. Vaccine 37, 3022-3030 (2019).

13. Gherardin, N.A. et al. Human blood MAIT cell subsets defined using MR1 tetramers. Immunol Cell Biol 96, 19 (2018).

14. Wang, H. et al. MAIT cells protect against pulmonary Legionella longbeachae infection. Nat Commun 9, 3350 (2018).

15. Le Bourhis, L. et al. MAIT cells detect and efficiently lyse bacterially-infected epithelial cells. PLoS Pathog 9, e1003681 (2013).

16. Hartmann, N. et al. Role of MAIT cells in pulmonary bacterial infection. Mol Immunol 101, 155-159 (2018). 
17. Meermeier, E.W., Harriff, M.J., Karamooz, E. \& Lewinsohn, D.M. MAIT cells and microbial immunity. Immunol Cell Biol 96, 607-617 (2018).

18. Suliman, S. et al. MR1-Independent Activation of Human Mucosal-Associated Invariant T Cells by Mycobacteria. J Immunol 203, 2917-2927 (2019).

19. Provine, N.M. et al. MAIT cell activation augments adenovirus vector vaccine immunogenicity. Science 371, 521-526 (2021).

20. Gherardin, N.A. et al. Diversity of T Cells Restricted by the MHC Class I-Related Molecule MR1 Facilitates Differential Antigen Recognition. Immunity 44, 32-45 (2016).

21. Le Nours, J. et al. A class of gammadelta T cell receptors recognize the underside of the antigen-presenting molecule MR1. Science 366, 1522-1527 (2019).

22. Reantragoon, R. et al. Antigen-loaded MR1 tetramers define T cell receptor heterogeneity in mucosal-associated invariant T cells. J Exp Med 210, 2305-2320 (2013).

23. Lepore, M. et al. Parallel T-cell cloning and deep sequencing of human MAIT cells reveal stable oligoclonal TCRbeta repertoire. Nat Commun 5, 3866 (2014).

24. Porcelli, S., Yockey, C.E., Brenner, M.B. \& Balk, S.P. Analysis of T cell antigen receptor (TCR) expression by human peripheral blood CD4-8- alpha/beta T cells demonstrates preferential use of several $\mathrm{V}$ beta genes and an invariant TCR alpha chain. J Exp Med 178, 1-16 (1993).

25. Gold, M.C. et al. MR1-restricted MAIT cells display ligand discrimination and pathogen selectivity through distinct T cell receptor usage. J Exp Med 211, 1601-1610 (2014).

26. Huang, $H$. et al. Select sequencing of clonally expanded CD8(+) T cells reveals limits to clonal expansion. Proc Natl Acad Sci U S A 116, 8995-9001 (2019).

27. Narayanan, G.A. et al. The MAIT TCRbeta chain contributes to discrimination of microbial ligand. Immunol Cell Biol (2020).

28. Harriff, M.J. et al. MR1 displays the microbial metabolome driving selective MR1restricted T cell receptor usage. Sci Immunol 3 (2018).

29. Eckle, S.B. et al. A molecular basis underpinning the T cell receptor heterogeneity of mucosal-associated invariant T cells. J Exp Med 211, 1585-1600 (2014).

30. Keller, A.N. et al. Drugs and drug-like molecules can modulate the function of mucosalassociated invariant T cells. Nat Immunol 18, 402-411 (2017).

31. Kjer-Nielsen, L. et al. MR1 presents microbial vitamin B metabolites to MAIT cells. Nature 491, 717-723 (2012).

32. Kjer-Nielsen, L. et al. An overview on the identification of MAIT cell antigens. Immunol Cell Biol 96, 573-587 (2018). 
33. Koay, H.F. et al. Diverse MR1-restricted T cells in mice and humans. Nat Commun 10, 2243 (2019).

34. Meermeier, E.W. et al. Human TRAV1-2-negative MR1-restricted T cells detect S. pyogenes and alternatives to MAIT riboflavin-based antigens. Nat Commun 7, 12506 (2016).

35. Gherardin, N.A., McCluskey, J., Rossjohn, J. \& Godfrey, D.I. The Diverse Family of MR1-Restricted T Cells. J Immunol 201, 2862-2871 (2018).

36. Awad, W. et al. Atypical TRAV1-2(-) T cell receptor recognition of the antigen-presenting molecule MR1. J Biol Chem 295, 14445-14457 (2020).

37. Crowther, M.D. et al. Genome-wide CRISPR-Cas9 screening reveals ubiquitous T cell cancer targeting via the monomorphic MHC class I-related protein MR1. Nat Immunol 21, 178-185 (2020).

38. Godfrey, D.I., Le Nours, J., Andrews, D.M., Uldrich, A.P. \& Rossjohn, J. Unconventional T Cell Targets for Cancer Immunotherapy. Immunity 48, 453-473 (2018).

39. Hinks, T.S. Mucosal-associated invariant T cells in autoimmunity, immune-mediated diseases and airways disease. Immunology 148, 1-12 (2016).

40. Newell, E.W. \& Davis, M.M. Beyond model antigens: high-dimensional methods for the analysis of antigen-specific T cells. Nat Biotechnol 32, 149-157 (2014).

41. Wang, G.C., Dash, P., McCullers, J.A., Doherty, P.C. \& Thomas, P.G. T cell receptor alphabeta diversity inversely correlates with pathogen-specific antibody levels in human cytomegalovirus infection. Sci Transl Med 4, 128ra142 (2012).

42. Glanville, J. et al. Identifying specificity groups in the T cell receptor repertoire. Nature 547, 94-98 (2017).

43. Dash, P. et al. Quantifiable predictive features define epitope-specific T cell receptor repertoires. Nature 547, 89-93 (2017).

44. Han, A., Glanville, J., Hansmann, L. \& Davis, M.M. Linking T-cell receptor sequence to functional phenotype at the single-cell level. Nat Biotechnol 32, 684-692 (2014).

45. Redmond, D., Poran, A. \& Elemento, O. Single-cell TCRseq: paired recovery of entire Tcell alpha and beta chain transcripts in T-cell receptors from single-cell RNAseq. Genome Med 8, 80 (2016).

46. Carlson, C.S. et al. Using synthetic templates to design an unbiased multiplex PCR assay. Nat Commun 4, 2680 (2013).

47. Zheng, G.X. et al. Massively parallel digital transcriptional profiling of single cells. Nat Commun 8, 14049 (2017). 
48. Elliott, J.I. \& Altmann, D.M. Dual T cell receptor alpha chain T cells in autoimmunity. $J$ Exp Med 182, 953-959 (1995).

49. Padovan, E. et al. Expression of two T cell receptor alpha chains: dual receptor T cells. Science 262, 422-424 (1993).

50. Schuldt, N.J. \& Binstadt, B.A. Dual TCR T Cells: Identity Crisis or Multitaskers? J Immunol 202, 637-644 (2019).

51. Suliman, S. et al. Peripheral blood mucosal-associated invariant T (MAIT) cells in tuberculosis patients and healthy Mycobacterium tuberculosis-exposed controls. $J$ Infect Dis (2020).

52. Lopez, K. et al. CD1b Tetramers Broadly Detect T Cells That Correlate With Mycobacterial Exposure but Not Tuberculosis Disease State. Front Immunol 11, 199 (2020).

53. Sant, S. et al. Single-Cell Approach to Influenza-Specific CD8(+) T Cell Receptor Repertoires Across Different Age Groups, Tissues, and Following Influenza Virus Infection. Front Immunol 9, 1453 (2018).

54. Nguyen, T.H. et al. Recognition of distinct cross-reactive virus-specific CD8+ T cells reveals a unique TCR signature in a clinical setting. J Immunol 192, 5039-5049 (2014).

55. Loh, L. et al. Human Mucosal-Associated Invariant T Cells in Older Individuals Display Expanded TCRalphabeta Clonotypes with Potent Antimicrobial Responses. J Immunol 204, 1119-1133 (2020).

56. Szymczak, A.L. et al. Correction of multi-gene deficiency in vivo using a single 'selfcleaving' 2A peptide-based retroviral vector. Nat Biotechnol 22, 589-594 (2004).

57. Howson, L.J. et al. MAIT cell clonal expansion and TCR repertoire shaping in human volunteers challenged with Salmonella Paratyphi A. Nat Commun 9, 253 (2018).

58. Malissen, M. et al. A T cell clone expresses two T cell receptor alpha genes but uses one alpha beta heterodimer for allorecognition and self MHC-restricted antigen recognition. Cell 55, 49-59 (1988).

59. Casanova, J.L., Romero, P., Widmann, C., Kourilsky, P. \& Maryanski, J.L. T cell receptor genes in a series of class I major histocompatibility complex-restricted cytotoxic T lymphocyte clones specific for a Plasmodium berghei nonapeptide: implications for $T$ cell allelic exclusion and antigen-specific repertoire. J Exp Med 174, 1371-1383 (1991).

60. Gascoigne, N.R. \& Alam, S.M. Allelic exclusion of the T cell receptor alpha-chain: developmental regulation of a post-translational event. Semin Immunol 11, 337-347 (1999).

61. Malissen, M. et al. Regulation of TCR alpha and beta gene allelic exclusion during T-cell development. Immunol Today 13, 315-322 (1992). 
62. Huang, C. \& Kanagawa, O. Ordered and coordinated rearrangement of the TCR alpha locus: role of secondary rearrangement in thymic selection. J Immunol 166, 2597-2601 (2001).

63. Dupic, T., Marcou, Q., Walczak, A.M. \& Mora, T. Genesis of the alphabeta T-cell receptor. PLoS Comput Biol 15, e1006874 (2019).

64. Tilloy, F. et al. An invariant T cell receptor alpha chain defines a novel TAP-independent major histocompatibility complex class Ib-restricted alpha/beta T cell subpopulation in mammals. J Exp Med 189, 1907-1921 (1999).

65. Exley, M., Garcia, J., Balk, S.P. \& Porcelli, S. Requirements for CD1d recognition by human invariant Valpha24+ CD4-CD8- T cells. J Exp Med 186, 109-120 (1997).

66. Van Rhijn, I. et al. A conserved human T cell population targets mycobacterial antigens presented by CD1b. Nat Immunol 14, 706-713 (2013).

67. van Schaik, B. et al. Discovery of invariant T cells by next-generation sequencing of the human TCR alpha-chain repertoire. J Immunol 193, 5338-5344 (2014).

68. Guo, J. et al. Regulation of the TCRalpha repertoire by the survival window of CD4(+)CD8(+) thymocytes. Nat Immunol 3, 469-476 (2002).

69. Schuldt, N.J. et al. Cutting Edge: Dual TCRalpha Expression Poses an Autoimmune Hazard by Limiting Regulatory T Cell Generation. J Immunol 199, 33-38 (2017).

70. Corthay, A., Nandakumar, K.S. \& Holmdahl, R. Evaluation of the percentage of peripheral $\mathrm{T}$ cells with two different $\mathrm{T}$ cell receptor alpha-chains and of their potential role in autoimmunity. J Autoimmun 16, 423-429 (2001).

71. Morris, G.P., Uy, G.L., Donermeyer, D., Dipersio, J.F. \& Allen, P.M. Dual receptor T cells mediate pathologic alloreactivity in patients with acute graft-versus-host disease. Sci Transl Med 5, 188ra174 (2013).

72. Kim, S.M. et al. Analysis of the paired TCR alpha- and beta-chains of single human T cells. PLoS One 7, e37338 (2012).

73. Koay, H.F. et al. A three-stage intrathymic development pathway for the mucosalassociated invariant T cell lineage. Nat Immunol 17, 1300-1311 (2016).

74. Legoux, F. et al. Molecular mechanisms of lineage decisions in metabolite-specific T cells. Nat Immunol 20, 1244-1255 (2019).

75. Koay, H.F., Godfrey, D.I. \& Pellicci, D.G. Development of mucosal-associated invariant T cells. Immunol Cell Biol 96, 598-606 (2018).

76. Egawa, T. et al. Genetic evidence supporting selection of the Valpha14i NKT cell lineage from double-positive thymocyte precursors. Immunity 22, 705-716 (2005).

77. Anderson, G. \& Takahama, Y. Thymic epithelial cells: working class heroes for T cell development and repertoire selection. Trends Immunol 33, 256-263 (2012). 
bioRxiv preprint doi: https://doi.org/10.1101/2021.03.25.436871; this version posted March 26, 2021. The copyright holder for this preprint

(which was not certified by peer review) is the author/funder, who has granted bioRxiv a license to display the preprint in perpetuity. It is made available under aCC-BY 4.0 International license.

78. Van Rhijn, I., Godfrey, D.I., Rossjohn, J. \& Moody, D.B. Lipid and small-molecule display by CD1 and MR1. Nat Rev Immunol 15, 643-654 (2015). 
A)

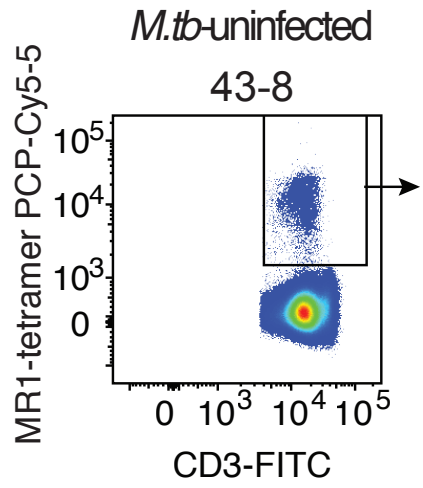

B)

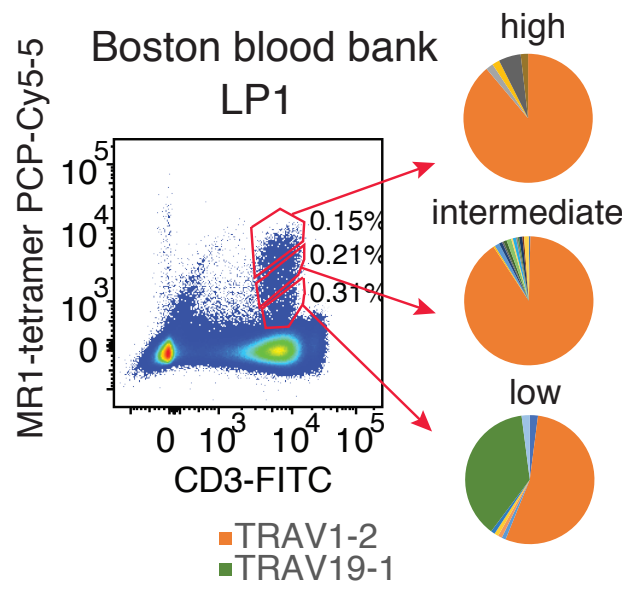

C)

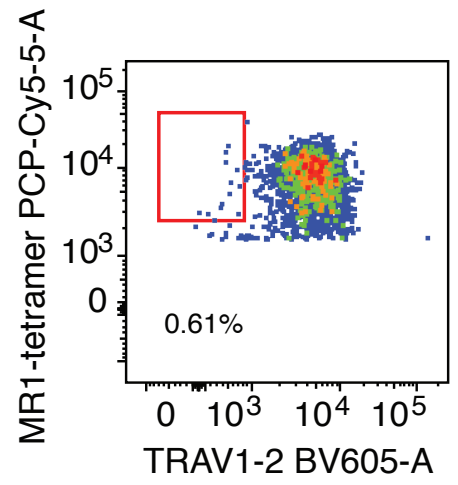

Active TB
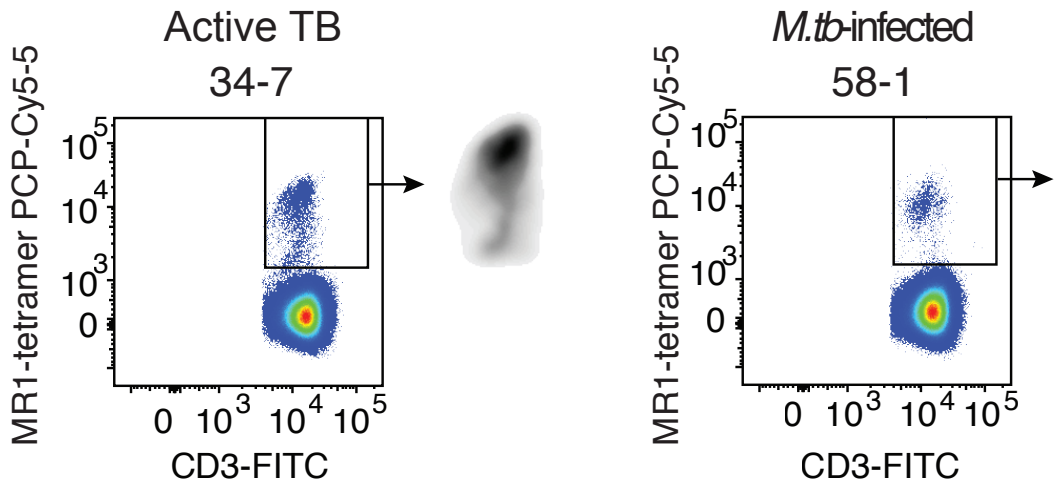

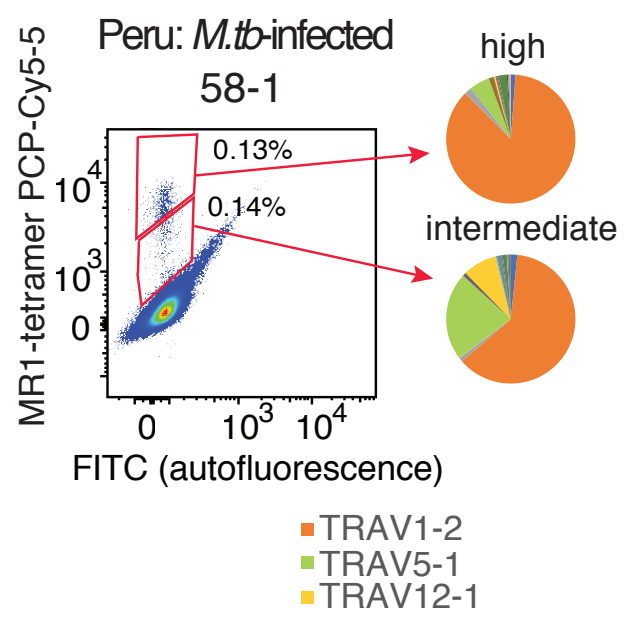

D)

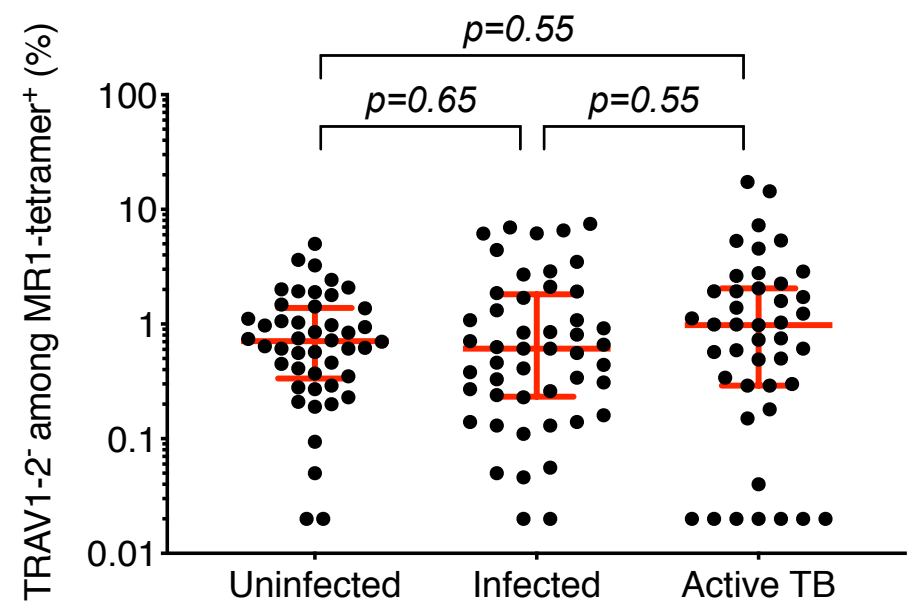


Figure 2

Suliman, et al

A) Peru:
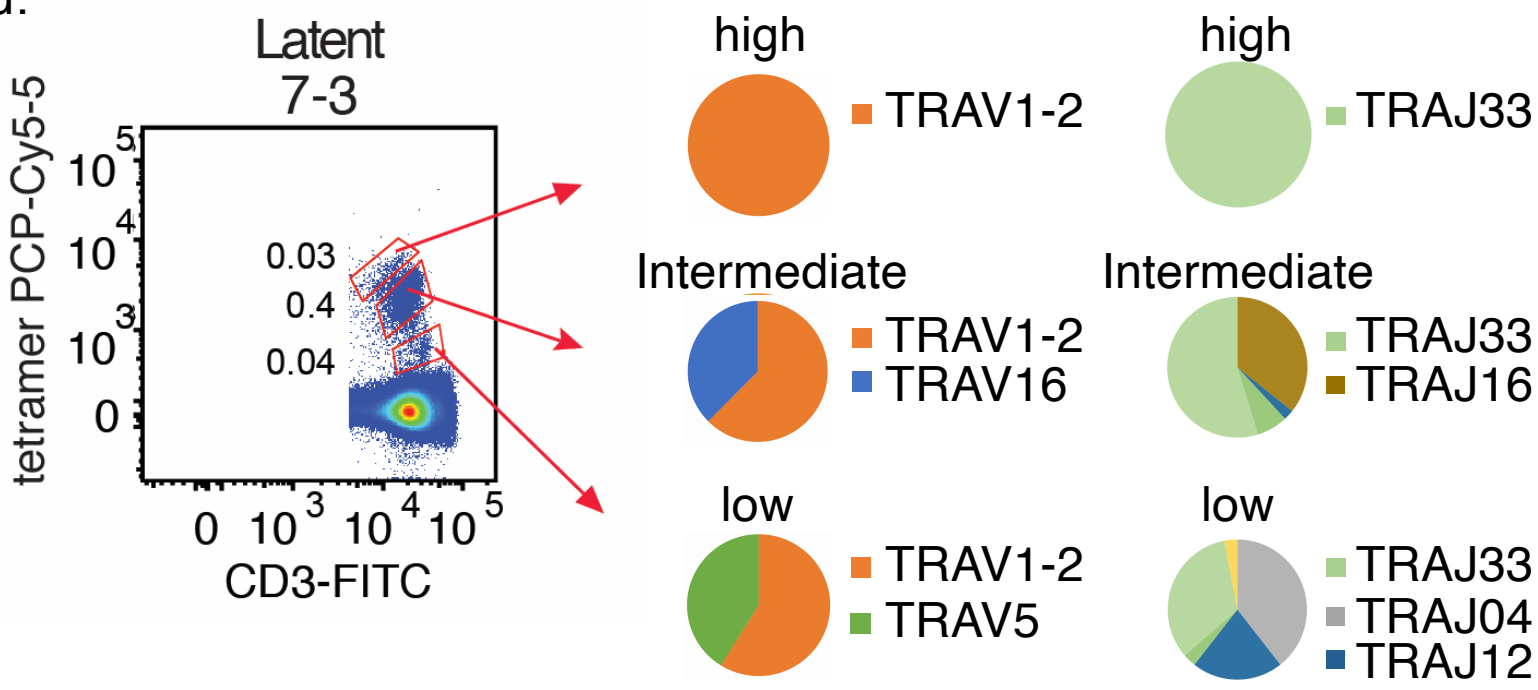

B) Boston blood bank:

\section{A 703A}

MR1-tetramer: high high

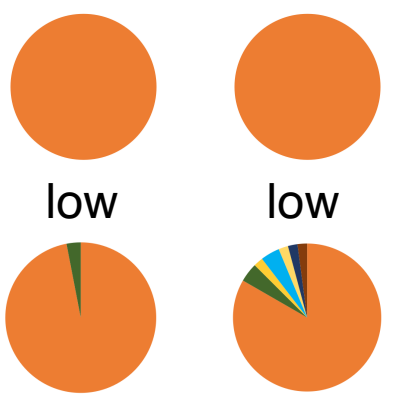

-TRAV1-2

- TRAV8-4 "TRAV23-1

-TRAV12-1 - TRAV26-1

- TRAV13-2 - TRAV26-2 


\begin{tabular}{|c|c|c|c|c|c|c|}
\hline $\begin{array}{l}\text { Tetramer } \\
\text { level }\end{array}$ & Donor & $\begin{array}{l}\text { Sort } \\
\text { Method }\end{array}$ & TCR & V-gene & $\begin{array}{l}\text { Complementarity } \\
\text { determining region (CDR3) }\end{array}$ & J-gene \\
\hline \multirow[b]{2}{*}{ Intermediate* } & \multirow{2}{*}{$\begin{array}{l}\text { Peru Latent } \\
7-3\end{array}$} & \multirow{2}{*}{$\begin{array}{l}\text { Single cell } \\
(\mathrm{PCR})\end{array}$} & $\mathrm{TCR} \alpha$ & TRAV16 & CALSGRRNSGYSTLTFGKG & TRAJ11 \\
\hline & & & TCR $\beta$ & TRBV24-1 & CATSDLGTDQETQYFGPG & TRBJ2-5 \\
\hline \multirow[b]{2}{*}{ low } & \multirow{2}{*}{$\begin{array}{l}\text { Peru Latent } \\
7-3\end{array}$} & \multirow{2}{*}{$\begin{array}{l}\text { Single cell } \\
(\mathrm{PCR})\end{array}$} & TCR $\alpha$ & TRAV5 & CAEAPGGYNKLIFGAG & TRAJ4 \\
\hline & & & TCR $\beta$ & TRBV6-1 & CASSEAAGTGGETQYFGPG & TRBJ2-5 \\
\hline \multirow[b]{2}{*}{ low } & \multirow{2}{*}{$\begin{array}{l}\text { Peru Latent } \\
58-1\end{array}$} & \multirow{2}{*}{$\begin{array}{l}\text { Bulk } \\
\text { (Adaptive) }\end{array}$} & $\mathrm{TCR} \alpha$ & TRAV5 & CAESSLDNYGQNFVF & TRAJ26 \\
\hline & & & TCR $\beta$ & TRBV15 & CATSKGSERSEQYF & TRBJ2-7 \\
\hline \multirow{2}{*}{ high } & \multirow{2}{*}{$\begin{array}{l}\text { Peru Latent } \\
58-1\end{array}$} & \multirow{2}{*}{$\begin{array}{l}\text { Bulk } \\
\text { (Adaptive) }\end{array}$} & $\mathrm{TCR} \alpha$ & TRAV1-2 & CAVPDSNYQLIW & TRAJ33 \\
\hline & & & TCR $\beta$ & TRBV601 & CASSEGPGTGHQPQHF & TRBJ1-5 \\
\hline
\end{tabular}



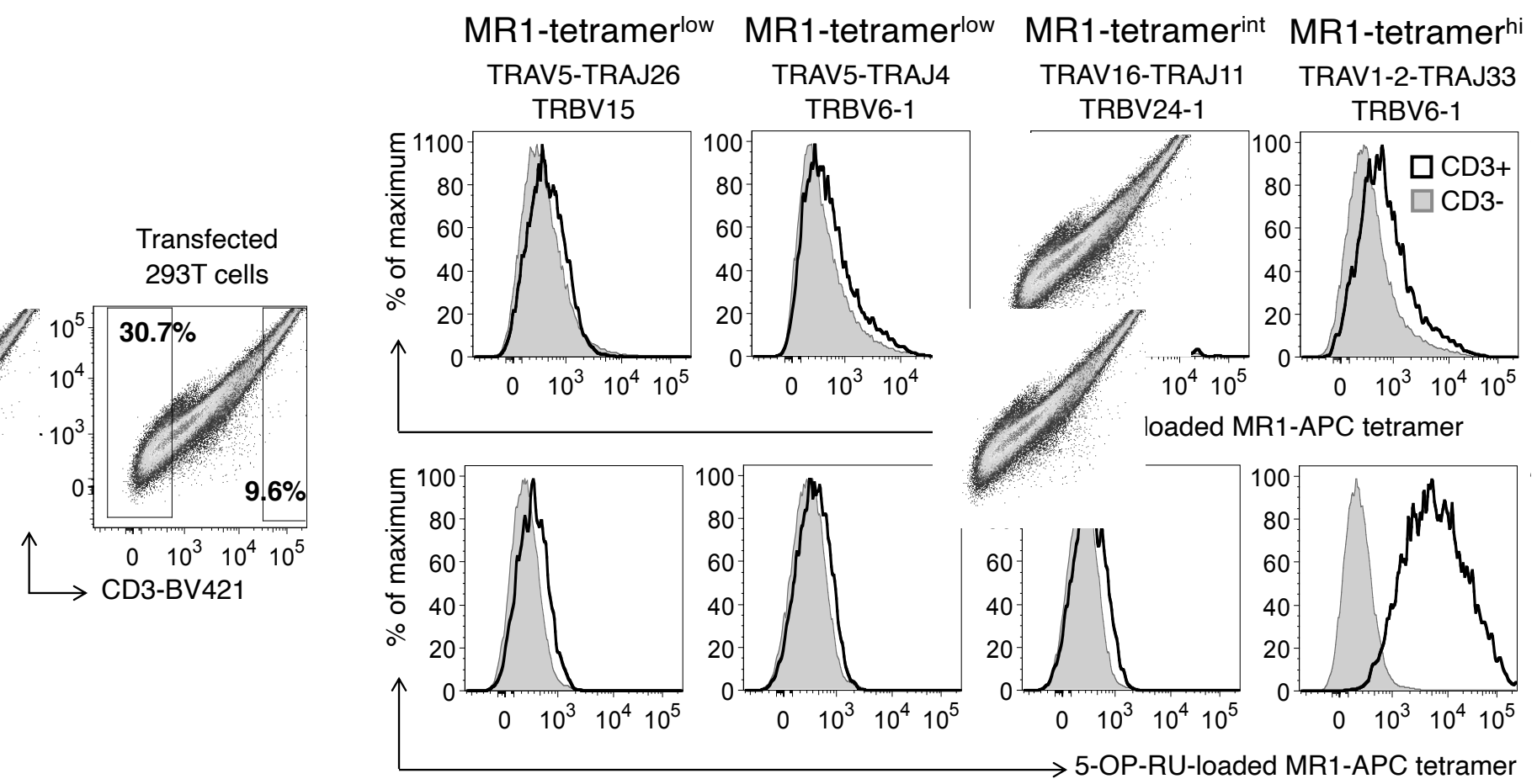
bioRxiv preprint doi: https://doi.org/10.1101/2021.03.25.436871; this version posted March 26, 2021. The copyright holder for this preprint (which was not certified by peer review) is the author/funder, who has granted bioRxiv a license to display the preprint in perpetuity. It is made available under aCC-BY 4.0 International license.

\section{Figure 5}

\section{Suliman, et al}

\begin{tabular}{|c|c|c|c|c|c|c|c|c|c|c|c|}
\hline \multirow{3}{*}{ 产 } & Donor & $\begin{array}{l}\text { Coded } \\
\text { Donor } \\
\text { name }\end{array}$ & TRAV & TRAJ & $\operatorname{CDR} 3 \alpha$ & TRAV & TRAJ & $\operatorname{CDR} 3 \alpha$ & TRBV & TRBJ & CDR3 $\beta$ \\
\hline & F3045C37 & $\mathrm{CH} 3$ & 6 & 9 & CALEHTGGFKTIF & $1-2$ & 33 & CAVSDSNYQLIW & $20-1$ & $2-1$ & CSARFASDYNEQFF \\
\hline & F2072 & $\mathrm{CH} 6$ & $1-2$ & 33 & CLVMDSNYQLIW & 16 & 33 & CALVDSNYQLIW & 6-4 & $2-3$ & CASSPTSGGATDTQYF \\
\hline \multirow{2}{*}{ 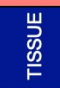 } & SP234 & SP2 & $1-2$ & 33 & CAAMDSNYQLIW & 16 & 16 & CALTVGDGQKLLF & 28 & $2-1$ & CASSRSGEYNEQFF \\
\hline & SP583 & SP1 & $1-2$ & 33 & CAVRDSNYQLIW & $8-2$ & 11 & CVVTLSPGYSTLTF & $4-2 / 4-3$ & $2-5$ & CASSPKTSGDIETQYF \\
\hline \multirow{4}{*}{ 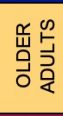 } & DMC12 & ED7 & $1-2$ & 33 & CAVRDSNYQLIW & 10 & 3 & CVVTLYSSASKIIF & $20-1$ & $1-6$ & CSARVGGPDSSPLHF \\
\hline & DMC12 & ED7 & $1-2$ & 33 & CAVMDSNYQLIW & $13-2$ & 8 & CAQEGFQKLVF & $29-1$ & $2-1$ & CSVGSPGQGGNEQFF \\
\hline & DMC15 & ED8 & $1-2$ & 33 & CAVRDSNYQLIW & 23/DV6 & 3 & CAASRNQIIF & $6-4$ & $2-1$ & CASSDGSGGNEQFF \\
\hline & DMC28 & ED33 & $1-2$ & 33 & CAATDSNYQLIW & $13-2$ & 16 & CAENSLLPISDGQKLLF & $4-2$ & $2-2$ & CASNQPTSGWTNTGELFF \\
\hline & KK5 & AD38 & $1-2$ & 33 & CAVMDSNYQLIW & $13-2$ & 27 & CAENANNTNAGKSTF & $20-1$ & $2-1$ & CSARLRDNEQFF \\
\hline
\end{tabular}

